\title{
Apoyo psicológico en desastres: Propuesta de un modelo de atención basado en revi- siones sistemáticas y metaanálisis
}

\author{
RODRIGO A. FIGUEROA ${ }^{1,2}$, HUMBERTO MARÍN³ \\ MATÍAS GONZÁLEZ ${ }^{2}$
}

\section{Psychological support for disaster victims: An evidence-based care model}

A search for meta analyses and systematic reviews on psychological support to disaster victims was carried out to devise a local support model. Based on 36 meta analyses and systematic reviews, the support should be carried out in five echelon levels: diffusion, social support, general medical care, general psychiatric care and psychiatric care carried out by experts. Only victims with well-established formal psychiatric disorders should receive psychotherapy or psychotropic medication. The rest should only receive psychological first aid. According to the best evidence available, a model for psychological care is proposed.

(Rev Med Chile 2010; 138: 143-151).

Key words: Disasters: Psychiatry; Psychology, medical.

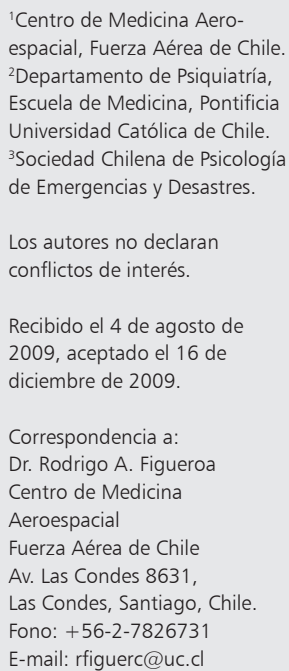

A ctualmente, el mundo se enfrenta a los desastres a una escala sin precedentes. Entre los años 1993 y 2003, los desastres cobraron cada año 58 mil vidas, afectaron a 255 millones de personas y costaron 67 billones de dólares. En el año 2003, una de cada 25 personas fue afectada por algún desastre. Desde la década 1950-59 el costo económico asociado a éstos se ha incrementado 14 veces. Las estimaciones indican que los desastres naturales podrían aumentar su frecuencia producto del cambio climático. El crecimiento poblacional, la urbanización y la pobreza podrían aumentar nuestra vulnerabilidad ${ }^{1}$.

Se considera desastre cualquier emergencia que sobrepase las capacidades de respuesta local, obligando a solicitar apoyo externo ${ }^{2}$. Para que una emergencia sea incluida en el Centro de Colaboración de la Organización Mundial de la Salud para el Estudio de la Epidemiología de los Desastres (Centre for Research on the Epidemiology of Disasters-CRED), ésta debe: a) Haber cobrado 10 o más vidas; b) Haber afectado a 100 o más personas; c) Haber obligado a declarar estado de emergencia; o d) Haber obligado a solicitar apoyo internacional' ${ }^{2}$.

Aunque hoy se sabe que la mayoría de los afectados por un desastre no desarrollará psicopatología, un grupo significativo lo haráa ${ }^{3-7}$. Dentro de las consecuencias psicológicas más frecuentes se encuentra el distrés subclínico ${ }^{3,4,8}$, el trastorno por estrés agudo-11, el trastorno por estrés post traumático (TEPT) ${ }^{3,4,12}$, la depresión mayor ${ }^{3,4}$, el aumento del consumo de alcohol y drogas ${ }^{13,14}$, otros trastornos de ansiedad ${ }^{4,15,16}$ y los síntomas de somatización ${ }^{17}$. Los factores moderadores de riesgo más importantes para el desarrollo de psicopatología, especialmente TEPT, son el nivel de percepción de amenaza vital, el nivel de apoyo social después del trauma, el tipo de respuesta emocional peri-traumática y el nivel de disociación peri-traumática ${ }^{18}$.

Aunque actualmente existen guías clínicas para el manejo de las víctimas de desastres ${ }^{19,20}$, éstas están escritas en inglés. La escasez de publi- 
caciones en nuestro idioma es preocupante. En una búsqueda en PubMed en abril de 2009, sólo encontramos 7 revisiones, metaanálisis o guías clínicas en castellano acerca de TEPT, comparado con 1.820 en inglés.

El objetivo de este trabajo es elaborar un modelo de intervención psicológica temprana en víctimas de desastre, escrito en castellano y fundamentado en la mejor evidencia disponible, de acuerdo a los principios de la medicina basada en la evidencia ${ }^{21}$.

\section{Materiales y Métodos}

Estrategia de búsqueda de evidencia: La búsqueda se limitó a revisiones sistemáticas y metaanálisis en cualquier idioma y fecha. Todas las búsquedas se efectuaron en diciembre de 2009.

Se efectuaron búsquedas en PubMed y en The Cochrane Database of Systematic Reviews con los términos "Stress Disorders, Traumatic" [Mesh] OR "Stress Disorders, Post-Traumatic" [Mesh] OR "Stress Disorders, Traumatic, Acute" [Mesh] Limits: Meta-Analysis.

Adicionalmente, se realizó una búsqueda amplia en Internet para encontrar evidencia acerca de la difusión de información durante los desastres y el apoyo social a las comunidades afectadas, ya que no encontramos metaanálisis que cubrieran estos temas.

Selección de la evidencia: Luego de revisar los resúmenes de todas las referencias encontradas, cada autor seleccionó las que podían relacionarse con la atención psicológica de víctimas de desastres. Las controversias fueron resueltas por acuerdo entre los autores. No se evaluó la calidad de las revisiones encontradas.

Elaboración de las recomendaciones: Luego de que todos los autores leyeran las referencias seleccionadas, se desarrolló en conjunto un modelo de respuesta, integrando la experiencia de cada autor con la evidencia encontrada.

\section{Resultados}

Se encontraron 78 revisiones sistemáticas o metaanálisis, sólo una en francés ${ }^{22}$ y dos en alemán ${ }^{23,24}$. De ellas se seleccionaron 36 para ser revisadas completamente ${ }^{22,56,63}$.

El siguiente modelo se circunscribe a la fase temprana de respuesta al desastre (primeros 3 meses), sin olvidar la importancia que tiene la preparación durante la fase de pre-impacto y la reconstrucción en la fase de post-impacto, lo que va más allá de los objetivos de este artículo. Deberá ser revisado periódicamente para su actualización, a la luz de la nueva evidencia científica.

\section{Tareas de la atención psicológica temprana}

Proponemos organizar la respuesta psicológica temprana en cinco niveles de atención: I. Difusión; II. Apoyo social; III. Manejo médico general; IV. Manejo psiquiátrico general; y V. Manejo psiquiátrico por expertos (Tabla 1). Cada uno de estos niveles se implementará en diferentes lugares físicos y tendrá distintos objetivos, beneficiarios, tareas, ejecutores y criterios de derivación al siguiente nivel, lo que determinará un flujo de pacientes desde centros de menor a mayor nivel de especialización (Figura 1). Toda la comunidad deberá ser informada acerca del desastre y recibir psicoeducación y oferta de atención psicológica a través de los medios de comunicación. La mayor parte de los afectados será atendido en los Centros de Afluencia de Público, como campamentos, albergues, escuelas, ollas comunes, etc. Un porcentaje menor será derivado a los Puestos Médicos Avanzados (Hospitales de Baja Complejidad u Hospitales de Campaña de las Fuerzas Armadas) para recibir atención médica general. Sólo aquellos que desarrollen trastornos psiquiátricos formales serán derivados a los Centros Psiquiátricos Generales. Los pacientes más graves, refractarios a los tratamientos basados en la evidencia, podrán ser sometidos a tratamientos de rescate en los Centros de Especialidad en TEPT. El objetivo es reservar la psicoterapia y farmacoterapia sólo para los pacientes con trastornos psiquiátricos formales, ya que es el único grupo que ha demostrado beneficiarse de estos tratamiento ${ }^{25,27}$. Para el resto de los afectados, incluyendo a aquellos con distrés subclínico, se recomienda limitar la atención a los llamados Primeros Auxilios Psicológicos ${ }^{57}$.

\section{Nivel I. Difusión}

Hoy se sabe que muchos de los afectados por desastres no reciben atención psicológica oportuna porque desconocen de su disponibilidad o 
Apoyo psicológico en desastres: Propuesta de un modelo de atención - R. A. Figueroa et al

porque no reconocen los primeros síntomas de los trastornos mentales ${ }^{58}$. Para evitar esto es necesario que toda la comunidad conozca la oferta de atención psicológica y los primeros síntomas que indican una patología psiquiátrica. Ello se podría lograr, en ambientes de desastre, a través de programas de psicoeducación en la televisión o la radio, así como desplegando panfletos en las cercanías del área de impacto. Las autoridades deberían designar un Oficial de Difusión encargado

Tabla 1. Respuesta psicológica a los desastres: Características de los distintos niveles de atención

\begin{tabular}{|c|c|c|c|c|c|c|}
\hline $\begin{array}{l}\text { Nivel de } \\
\text { atención }\end{array}$ & Objetivos & Beneficiarios & $\begin{array}{c}\text { Tareas } \\
\text { específicas }\end{array}$ & Ejecutores & Lugar físico & $\begin{array}{c}\text { Criterios de } \\
\text { derivación } \\
\text { al siguiente } \\
\text { nivel }\end{array}$ \\
\hline $\begin{array}{l}\text { Nivel I } \\
\text { Difusión }\end{array}$ & $\begin{array}{l}\text { - Mejorar la } \\
\text { cobertura } \\
\text { - Psicoeducar } \\
\text { - Disminuir la } \\
\text { incertidum- } \\
\text { bre }\end{array}$ & $\begin{array}{l}\text { - Toda la } \\
\text { comunidad }\end{array}$ & $\begin{array}{l}\text { - Psicoeduca- } \\
\text { ción y oferta } \\
\text { de atención a } \\
\text { través de los } \\
\text { medios } \\
\text { - Comunicados } \\
\text { oficiales }\end{array}$ & $\begin{array}{l}\text { - Oficial de } \\
\text { Difusión } \\
\text { - Medios de } \\
\text { comunica- } \\
\text { ción }\end{array}$ & $\begin{array}{l}\text { - Área de } \\
\text { impacto y } \\
\text { alrededores }\end{array}$ & \\
\hline $\begin{array}{l}\text { Nivel II } \\
\text { Apoyo social }\end{array}$ & $\begin{array}{l}\text { - Facilitar la } \\
\text { recuperación } \\
\text { espontánea y } \\
\text { resiliencia } \\
\text { - Identificar y } \\
\text { derivar a los } \\
\text { afectados con } \\
\text { emergencias } \\
\text { psiquiátricas } \\
\text { o en riesgo } \\
\text { de desarrollar } \\
\text { un trastorno } \\
\text { psiquiátrico }\end{array}$ & $\begin{array}{l}\text { - Población } \\
\text { concentrada } \\
\text { en Centros } \\
\text { de Afluencia } \\
\text { de Público }\end{array}$ & $\begin{array}{l}\text { - Primeros Auxi- } \\
\text { lios Psicoló- } \\
\text { gicos } \\
\text { - Screening } \\
\text { Psicológico }\end{array}$ & $\begin{array}{l}\text { - Trabajadores } \\
\text { sociales, } \\
\text { coordinados } \\
\text { y asesora- } \\
\text { dos por un } \\
\text { psicólogo } \\
\text { entrenado } \\
\text { en Primeros } \\
\text { Auxilios } \\
\text { Psicológicos }\end{array}$ & $\begin{array}{l}\text { - Centros de } \\
\text { Afluencia } \\
\text { de Público }\end{array}$ & $\begin{array}{l}\text { - Uno o más } \\
\text { criterios del } \\
\text { screening } \\
\text { psicológico } \\
\text { positivo }\end{array}$ \\
\hline $\begin{array}{l}\text { Nivel III } \\
\text { Manejo } \\
\text { médico } \\
\text { general }\end{array}$ & $\begin{array}{l}\text { - Estabilizar los } \\
\text { síntomas } \\
\text { - Efectuar el } \\
\text { diagnóstico } \\
\text { psiquiátrico } \\
\text { - Derivar al } \\
\text { manejo psi- } \\
\text { quiátrico }\end{array}$ & $\begin{array}{l}\text { - Afectados } \\
\text { con síntomas } \\
\text { intensos o } \\
\text { en riesgo de } \\
\text { desarrollar } \\
\text { un trastorno } \\
\text { psiquiátrico }\end{array}$ & $\begin{array}{l}\text { - Farmacoterapia } \\
\text { ansiolítica } \\
\text { - Diagnóstico } \\
\text { psiquiátrico }\end{array}$ & $\begin{array}{l}\text { - Médicos } \\
\text { generales y } \\
\text { de urgencia }\end{array}$ & $\begin{array}{l}\text { - Puesto } \\
\text { Médico } \\
\text { Avanzado }\end{array}$ & $\begin{array}{l}\text { - TEPT, DM o } \\
\text { TEA } \\
\text { - Necesidad de } \\
\text { hospitaliza- } \\
\text { ción }\end{array}$ \\
\hline $\begin{array}{l}\text { Nivel IV } \\
\text { Manejo } \\
\text { psiquiátrico } \\
\text { general }\end{array}$ & $\begin{array}{l}\text { - Tratar los } \\
\text { trastornos } \\
\text { psiquiátricos } \\
\text { establecidos }\end{array}$ & $\begin{array}{l}\text { - Pacientes con } \\
\text { TEA, TEPT, } \\
\text { DM, etc. }\end{array}$ & $\begin{array}{l}\text {-PCT (TCC o } \\
\text { EMDR) } \\
\text { • Farmacoterapia }\end{array}$ & $\begin{array}{l}\text { - Terapeutas } \\
\text { con entre- } \\
\text { namiento en } \\
\text { PCT y farma- } \\
\text { coterapia }\end{array}$ & $\begin{array}{l}\text { - Centros Psi- } \\
\text { quiátricos } \\
\text { Generales }\end{array}$ & $\begin{array}{l}\text { - Refratarie- } \\
\text { dad a los } \\
\text { tratamientos } \\
\text { basados en la } \\
\text { evidencia }\end{array}$ \\
\hline $\begin{array}{l}\text { Nivel V } \\
\text { Manejo } \\
\text { psiquiátrico } \\
\text { por expertos }\end{array}$ & $\begin{array}{l}\text { - Tratar a los } \\
\text { pacientes } \\
\text { refractarios }\end{array}$ & $\begin{array}{l}\text { - Pacientes } \\
\text { refractarios }\end{array}$ & $\begin{array}{l}\text { - Psicoterapias } \\
\text { experimentales } \\
\text { - Farmacotera- } \\
\text { pias experi- } \\
\text { mentales }\end{array}$ & $\begin{array}{l}\text { - Terapeutas } \\
\text { expertos en } \\
\text { TEPT y otros } \\
\text { trastornos } \\
\text { relacionados }\end{array}$ & $\begin{array}{l}\text { - Centro de } \\
\text { especia- } \\
\text { lidad en } \\
\text { TEPT }\end{array}$ & \\
\hline
\end{tabular}

DM: Depresión Mayor; EMDR: Eye Movement Desensitization and Reprocessing; PCT: Psicoterapia Centrada en el Trauma; TCC: Terapia Cognitivo-Conductual; TEA: Trastorno por Estrés Agudo; TEPT: Trastorno por Estés Post Traumático. 


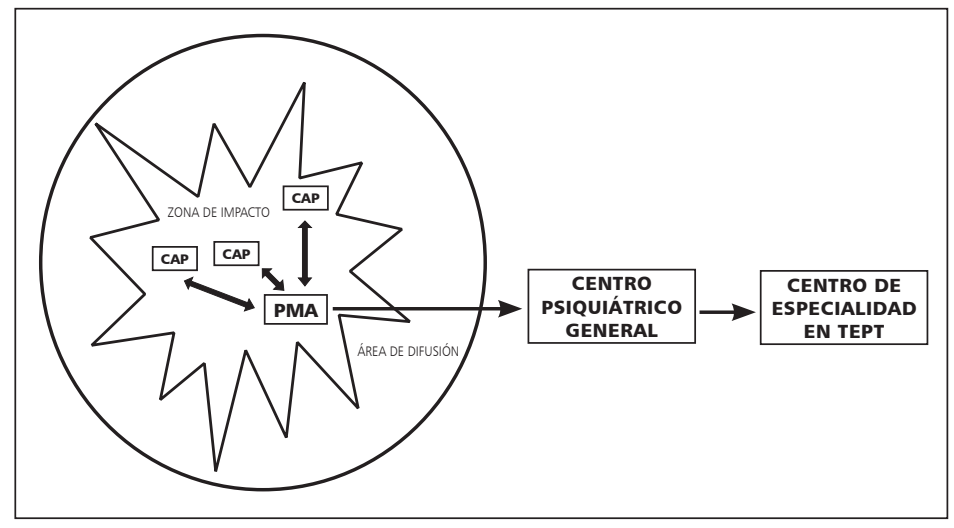

Figura 1. Respuesta psicológica a los desastres: Flujo de pacientes. CAP: Centro de afluencia de público (ollas comunes, campamentos, alberues, etc); PMA: Puesto médico avanzado (Hospital de baja complejidad u Hospital de campaña FF.AA.); TEPT: Trastorno por estrés post traumático. exclusivamente de estas funciones. Una adecuada coordinación entre el Oficial de Difusión y los medios de comunicación es indispensable ${ }^{59}$.

Además de aumentar la cobertura de atención psicológica, otro objetivo de la difusión es informar a la población acerca de la evolución del desastre. Esta información debe ser oportuna, precisa y deberá provenir de una única fuente (autoridades oficiales) para evitar contradicciones que aumenten la incertidumbre. La información retrasada, imprecisa o contradictoria podría aumentar la revictimización ${ }^{60}$. Minimizar los alcances del desastre para evitar el pánico de masas no está justificado, ya que las observaciones de campo indican que dicho fenómeno es excepcional y la minimización podría perjudicar la confianza de la población en sus autoridades, generando caos y descontento social ${ }^{61}$.

\section{Nivel II. Apoyo social}

En vista de que no hay evidencia de que la psicoterapia o la farmacoterapia beneficie a todos los afectados por un desastre ${ }^{25,27}$, la atención a la población ubicada en los Centros de Afluencia de Público debería limitarse a brindar seguridad y confort, monitorizar el estado emocional, facilitar el retorno a las rutinas y orientar en el uso de los recursos disponibles, lo que se conoce como Primeros Auxilios Psicológicos ${ }^{57}$ (Tabla 2). Éstos recomiendan no forzar a los afectados a hablar de sus sentimientos, ya que las intervenciones psicoterapéuticas que lo hacen, incluyendo el debreafing, no han demostrado reducir el desarrollo de trastornos psiquiátricos posteriores y peor aún podrían aumentarlos s $^{43,4-49,56}$.
Otro objetivo del apoyo social será identificar a los afectados en riesgo de desarrollar trastornos psiquiátricos o a aquellos que presenten alguna emergencia psiquiátrica, para derivarlos a la atención médica. Este proceso se conoce como screening psicológico o triage psicológico y se han desarrollado múltiples instrumentos para realizarlo $^{55}$. En general, los instrumentos de screening cortos y sencillos han demostrado ser tan o más efectivos que los largos y complejos. Nosotros recomendamos utilizar el Disaster-Related Psychological Screening Test (DRPST) ${ }^{55}$ por su simpleza ( 7 ítems, punto de corte $>=3$ ), su efectividad (sensibilidad 0,98; especificidad 0,97 para TEPT) y por ser uno de los pocos instrumentos validados en una población de víctimas de desastres. Combinado con la pesquisa de emergencias psiquiátricas, ofrece una excelente herramienta de screening para seleccionar a quiénes derivar a la atención médica general (Tabla 3).

Tanto los Primeros Auxilios Psicológicos como el screening psicológico deberían ser implementados en cada uno de los Centros de Afluencia de Público por los trabajadores sociales provenientes de organizaciones de asistencia humanitaria (Defensa Civil, organizaciones no gubernamentales, Cruz Roja, etc), coordinados por un psicólogo especialmente entrenado para ello.

\section{Nivel III. Manejo médico general}

La población derivada desde los Centros de Afluencia de Público debería recibir atención médica general con tres objetivos: $1^{\circ}$ Estabilizar los síntomas psicológicos invalidantes; $2^{\circ}$ Efectuar el diagnóstico psiquiátrico y $3^{\circ}$ Derivar al manejo 
Apoyo psicológico en desastres: Propuesta de un modelo de atención - R. A. Figueroa et al

Tabla 2. Primeros Auxilios Psicológicos

\begin{tabular}{|ll|}
\hline Acciones principales & Objetivos \\
\hline Contacto y encuentro & $\begin{array}{l}\text { Responder a los encuentros iniciados por los afectados o iniciar el encuentro } \\
\text { de un modo no invasivo, asistencial y compasivo }\end{array}$ \\
\hline Seguridad y confort & $\begin{array}{l}\text { Promover la seguridad inmediata y posterior y proveer confort físico y emo- } \\
\text { cional }\end{array}$ \\
\hline Estabilización & $\begin{array}{l}\text { Tranquilizar y orientar emocionalmente a los afectados sobrepasados o des- } \\
\text { orientados }\end{array}$ \\
\hline inmediatas y preocupaciones & $\begin{array}{l}\text { Identificar las necesidades inmediatas y las preocupaciones, recopilar informa- } \\
\text { ción adicional y adaptar las intervenciones de los Primeros Auxilios Psicológicos } \\
\text { a la realidad de cada paciente }\end{array}$ \\
\hline Asistencia práctica & $\begin{array}{l}\text { Ofrecer ayuda práctica a los afectados en la satisfacción de sus necesidades } \\
\text { inmediatas y la resolución de sus preocupaciones }\end{array}$ \\
\hline Información en afrontamiento & $\begin{array}{l}\text { Ayudar a lograr el contacto inmediato o posterior con las redes de apoyo } \\
\text { cercanas y otras fuentes de ayuda, incluyendo la familia, amigos y recursos } \\
\text { asistenciales de la comunidad }\end{array}$ \\
\hline Vinculación con servicios colaborativos & $\begin{array}{l}\text { Informar acerca de las reacciones de estrés y afrontamiento, para aliviar el } \\
\text { estrés y promover el funcionamiento adaptativo }\end{array}$ \\
\hline
\end{tabular}

Tabla 3. Screening Psicológico*

Emergencia psiquiátrica

Riesgo de agresión a terceros o sí mismo

Ideas delirantes o alucinaciones

Síntomas psicológicos invalidantes

(Ej. Crisis de pánico, insomnio pertinaz)

Puntaje DRPST $\geq 3$

* Derivar al nivel III si existe cualquier emergencia psiquiátrica o si el puntaje del DRPST es $\geq 3$. DRPST: Disaster-Related Psychological Screening Test.

psiquiátrico general si corresponde. Estas acciones deberían ser efectuadas por médicos generales y de urgencia en Puestos Médicos Avanzados (hospitales de baja complejidad u hospitales de campaña de las Fuerzas Armadas). En la Tabla 1 se detallan los criterios de derivación sugeridos.

Para la estabilización de los síntomas psicológicos invalidantes, como insomnio pertinaz, irritabilidad marcada, crisis de pánico, crisis de angustia, etc, recomendamos el uso de benzodia- zepinas, aunque no hayan demostrado ser efectivas para prevenir el desarrollo de psicopatología posterior ${ }^{35,38}$. Por su bajo riesgo de efectos adversos, su rápida acción y su efecto sostenido, sugerimos el uso de benzodiazepinas de vida media larga, como diazepam o clonazepam. No recomendamos el uso de antipsicóticos por el mayor riesgo de efectos adversos (distonías, síntomas extrapiramidales, síndrome neuroléptico maligno, etc).

Para apoyar el proceso diagnóstico, recomendamos utilizar los criterios diagnósticos del DSM-IV ${ }^{62}$ (Tabla 4). Puesto que se ha demostrado que los pacientes con trastorno por estrés agudo pueden reducir el riesgo de desarrollar TEPT si reciben psicoterapia entre las dos y cuatro semanas posteriores al trauma ${ }^{25,27}$, todos los pacientes que lleguen al nivel III deberían ser controlados dos semanas después del trauma, para implementar inmediatamente la psicoterapia si se diagnostica un trastorno por estés agudo en el control.

\section{Nivel IV. Manejo psiquiátrico general}

Por la necesidad de aplicar técnicas que requieren un entrenamiento especial y que se desarrolla a los largo de múltiples sesiones, la atención de los pacientes con trastornos psiquiátricos traumáticos debería ser efectuada por profesionales bien entre- 
Apoyo psicológico en desastres: Propuesta de un modelo de atención - R. A. Figueroa et al

\section{Tabla 4. Síntomas de TEPT}

EI TEPT se caracteriza por la presencia de tres grupos de síntomas que deben estar presentes por al menos un mes. Se requiere al menos un síntoma del grupo reexperimentación, tres síntomas del grupo evitación y embotamiento emocional y dos síntomas del grupo hiperalerta

Re-experimentación-recuerdos intrusivos y angustiantes del evento traumático; flashbacks; pesadillas; reacciones físicas y psicológicas intensas y molestas, como sudoración, palpitaciones o crisis de pánico al enfrentarse a personas, lugares o cosas que recuerden el trauma

Evitación y embotamiento emocional-evitación de actividades, lugares, pensamientos, sentimientos o conversaciones relacionadas al evento; restricción de las emociones; pérdida del interés en las actividades normales; sentimientos de desapego con los demás; amnesia psicógena; sensación de que no hay futuro

Hiperalerta-insomnio; irritabilidad; dificultades para concentrarse; hipervigilancia; sobresaltos

nados y en lugares que permitan la continuidad del tratamiento ${ }^{36}$. Proponemos que sean efectuadas en centros psiquiátricos generales, por ejemplo, hospitales de día. En aquellos casos que exista una emergencia psiquiátrica, debería considerarse la internación del paciente.

Aunque se ha demostrado que tanto la psicoterapia como la farmacoterapia son útiles en el tratamiento del TEPT ${ }^{25,27,31,38}$, la evidencia actual indica que las psicoterapias son más eficaces que la farmacoterapia y presentan un menor abandono de tratamiento ${ }^{26}$, por lo que son la primera línea de tratamiento. Además, en caso de que la farmacoterapia se inicie desde un comienzo, la psicoterapia podría potenciar su efecto ${ }^{45}$. De todas las psicoterapias, las psicoterapias individuales centradas en el trauma son las que tienen una mayor evidencia de efectividad $23,31,34,40,44,46,52,63$. Otras psicoterapias que también han demostrado ser efectivas, aunque menos que las anteriores, son las técnicas de manejo del estrés y la psicoterapia conductual grupal ${ }^{31,34,51,63}$. Actualmente, las psicoterapias centradas en el trauma pueden implementarse en dos formas: terapia cognitivoconductual o eye movement desesitization and reprocessing (EMDR). Ambas consisten esencialmente en exposición sistemática, desensibiliza- ción y reestructuración cognitiva. En el caso de EMDR, se utilizan los movimientos oculares para facilitar este proceso, pero la utilidad de ello es controvertida $^{37,44,52}$. La evidencia, por el contrario, indica que sería el componente conductual, común a todas las psicoterapias centradas en el trauma, el que explicaría su efecto benéfico ${ }^{42}$ y no se han encontrado diferencias de efectividad entre la terapia cognitivo-conductual y EMDR en el tratamiento del TEPT ${ }^{37}$. Las psicoterapias sin un componente conductual, como las psicoterapias de apoyo, la hipnosis, la psicoterapia psicodinámica, etc, no han demostrado ser efectivas ${ }^{31,34,40,63}$; sólo dos metaanálisis plantean lo contrario ${ }^{29,46}$. En el caso de EMDR, se han observado efectos benéficos incluso después de 15 meses de completada la terapia $^{52}$. Las psicoterapias centradas en el trauma también se pueden aplicar en niños, en quienes han mostrado una efectividad moderada ${ }^{51}$, ya sea en forma individual o grupal ${ }^{50,51}$. Otras psicoterapias en niños y adolescentes no tienen evidencia suficiente ${ }^{51}$.

Cuando el paciente no ha respondido a la psicoterapia, cuando se rehúsa a participar de ella, cuando la psicoterapia está contraindicada o si existe depresión mayor ${ }^{35}$, se debería considerar el uso de psicofármacos. La evidencia actual apoya el uso inicial de inhibidores selectivos de la recaptura de serotonina ${ }^{26,35,38}$ (ISRS), de los cuales la sertralina es el único que cuenta con metaanálisis que avalan su efectividad ${ }^{53,54}$. Los tratamientos deberían mantenerse por un período largo, en nuestra experiencia, hasta completar al menos un año de remisión de síntomas ${ }^{35}$. Otros antidepresivos más nuevos, los antidepresivos tricíclicos o la carbamazepina se podrían intentar cuando los ISRS hayan fallado, así como el tratamiento ansiolítico con benzodiazepinas ${ }^{26,35,38}$.

\section{Nivel V. Manejo psiquiátrico por expertos}

Aunque la evidencia indica que la efectividad de los inhibidores de la mono-amino-oxidasa (IMAO) y los antidepresivos tricíclicos es similar ${ }^{26}$, los IMAO sólo deberían ser utilizados cuando otros antidepresivos han fallado, por su alto riesgo de efectos adversos severos. La adición de olanzapina y risperidona podría ser útil, especialmente en los síntomas de intrusión ${ }^{28}$. El ácido valproico también podría ser útili ${ }^{33}$. En cualquier caso, estos y otros tratamientos experimentales, como la realidad virtual ${ }^{30}$, deberían ser indicados por ex- 
Apoyo psicológico en desastres: Propuesta de un modelo de atención - R. A. Figueroa et al

pertos, sólo en los casos más graves e idealmente en centros de especialidad en TEPT.

\section{Discusión}

Los desastres podrían aumentar en los próximos años, como consecuencia de los cambios climáticos, la sobrepoblación en zonas de riesgo y las acciones terroristas ${ }^{1}$. Ello aumenta la importancia de desarrollar estrategias para paliar sus consecuencias.

Aunque la mayoría de las víctimas de los desastres presenta síntomas psicológicos transitorios, sólo una minoría desarrolla psicopatología de largo plazo, siendo el TEPT y la depresión mayor las enfermedades más frecuentes. A pesar de la disponibilidad, la mayoría de los afectados por los desastres no pide ayuda en los servicios de salud mental ${ }^{58}$, por lo que luego de un desastre la demanda no se incrementa significativamente. Es necesario implementar estrategias de difusión de la salud mental para reducir el impacto del desastre sobre la población.

En la fase inmediata post-desastre, se recomienda asistir a las víctimas asegurando la cobertura de necesidades básicas, como método más efectivo para prevenir la aparición de psicopatología, lo que se conoce como Primeros Auxilios Psicológicos. En fases intermedias y luego de un proceso de screening psicológico que identifique a las víctimas en riesgo de desarrollar psicopatología grave, se recomienda aplicar técnicas preventivas y curativas. De ellas, las más avaladas por la evidencia son las psicoterapias centradas en el trauma.

Es sorprendente la escasez de publicaciones en castellano acerca de desastres. La comunidad científica hispanoparlante debería reforzar sus esfuerzos por desarrollar investigación en esta área y publicar en nuestro idioma. La falta de literatura es preocupante, porque podría aumentar nuestra vulnerabilidad a los desastres. Es necesario además entrenar al personal en Primeros Auxilios Psicológicos, ya que la falta de profesionales sociales entrenados en esta técnica podría afectar gravemente la capacidad de respuesta de nuestras comunidades.

Este trabajo tiene dos limitaciones importantes. En primer lugar, la cantidad de fuentes de información utilizadas fue limitada, por lo que se puede haber omitido trabajos importantes. Se- gundo, no se evaluó la calidad de las publicaciones seleccionadas. Todo esto podría comprometer la validez de nuestras recomendaciones, sin embargo, el gran tamaño de las dos bases de datos que utilizamos nos permite suponer que no deben ser muchos los metaanálisis omitidos. Además, la similitud de resultados entre los distintos metaanálisis que encontramos nos permite pensar que es poco probable que los trabajos omitidos puedan cambiar significativamente nuestras conclusiones.

\section{Referencias}

1. Guha-Sapir D, Hargitt D, Hoyois P. Thirty years of natural disasters 1993-2003: The numbers. 1st ed. Louvain-la Neuve: Presses Universitaires de Louvain; 2004.

2. Emergency Events Database (EM-DAT). Centre for Research on the Epidemiology of Disasters (CRED). Available from: http://www.emdat.be/ExplanatoryNotes/ glossary.html. Accessed 29 April 2009.

3. Galea S, Ahern J, Resnick H, Kilpatrick D, Bucuvalas M, Gold J, et al. Psychological sequelae of the September 11 terrorist attacks in New York City. N Engl J Med 2002; 346: 982-7.

4. North CS, Nixon SJ, Shariat S, Mallonee S, McMillen JC, Spitznagel EL, et al. Psychiatric disorders among survivors of the Oklahoma City bombing. JAMA. 1999; 282: 755-62.

5. Schlenger WE, Caddell JM, Ebert L, Jordan BK, Rourke $\mathrm{KM}$, Wilson D, et al. Psychological reactions to terrorist attacks: findings from the National Study of Americans' Reactions to September 11. JAMA 2002; 288: 581-8.

6. Schuster MA, Stein BD, Jaycox L, Collins RL, Marshall $\mathrm{GN}$, Elliott $\mathrm{MN}$, et al. A national survey of stress reactions after the September 11, 2001, terrorist attacks. N Engl J Med 2001; 345: 1507-12.

7. Silver RC, Holman EA, McIntosh DN, Poulin M, GilRivas V. Nationwide longitudinal study of psychological responses to September 11. JAMA 2002; 288: 1235-44.

8. North CS, Pfefferbaum B. Research on the mental health effects of terrorism. JAMA 2002; 288: 633-6.

9. Creamer M, Manning C. Acute stress disorder following an industrial accident. Australian Psychologist 1998; 33: 125-9.

10. Classen C, Koopman C, Hales R, Spiegel D. Acute stress disorder as a predictor of posttraumatic stress symptoms. Am J Psychiatry 1998; 155: 620-4.

11. Brewin CR, Andrews B, Rose S, Kirk M. Acute stress disorder and posttraumatic stress disorder in victims of violent crime. Am J Psychiatry 1999; 156: 360-6. 
12. Kessler RC, Sonnega A, Bromet E, Hughes M, Nelson CB. Posttraumatic stress disorder in the National Comorbidity Survey. Arch Gen Psychiatry 1995; 52: 1048-60.

13. Vlahov D, Galea S, Resnick H, Ahern J, Boscarino JA, Bucuvalas M, et al. Increased use of cigarettes, alcohol, and marijuana among Manhattan, New York, residents after the September 11th terrorist attacks. Am J Epidemiol 2002; 155: 988-96.

14. Pfefferbaum B, Doughty DE. Increased alcohol use in a treatment sample of Oklahoma City bombing victims. Psychiatry 2001; 64: 296-303.

15. David D, Mellman TA, Mendoza LM, Kulick-Bell R, Ironson G, Schneiderman N. Psychiatric morbidity following Hurricane Andrew. J Trauma Stress 1996; 9: 607-12.

16. North CS, Smith EM, Spitznagel EL. Posttraumatic stress disorder in survivors of a mass shooting. Am J Psychiatry 1994; 151: 82-8.

17. North CS. Epidemiology of disaster mental health. In: Ursano RJ, Fullerton CS, Weisaeth L, Raphael B, eds. Textbook of Disaster Psychiatry. 1st ed. New York: Cambridge University Press; 2007. Pp. 29-47.

18. Ozer EJ, Best SR, Lipsey TL, Weiss DS. Predictors of posttraumatic stress disorder and symptoms in adults: a meta-analysis. Psychol Bull 2003; 129: 52-73.

19. Forbes D, Creamer M, Phelps A, Bryant R, McFarlane A, Devilly GJ, et al. Australian guidelines for the treatment of adults with acute stress disorder and post-traumatic stress disorder. Aust N Z J Psychiatry 2007; 41: 637-48.

20. National Institute for Clinical Excellence (NICE). Posttraumatic stress disorder: the management of PTSD in adults and children in primary and secondary care. London; 2005.

21. Guyatt GH, Naylor D, Richardson WS, Green L, Haynes $\mathrm{RB}$, Wilson MC, et al. What is the best evidence for making clinical decisions? JAMA 2000; 284: 3127-8.

22. Aulagnier M, Verger P, Rouillon F. Efficacité du débriefing psychologique pour prévenir le stress post-traumatique. Rev Epidemiol Sante Publique 2004; 52: 67-79.

23. Sack M, Lempa W, Lamprecht F. Metaanalyse der Studien zur EMDR-Behandlung von Patienten mit posttraumatischen Belastungstörungen. Psychother Psychosom Med Psychol 2001; 51: 350-5.

24. Mitte K, Steil R, Nachtigall C. Eine Meta-Analyse unter Einsatz des Random Effects-Modells zur Effektivität kurzfristiger psychologischer Interventionen nach akuter Traumatisierung. Zeitschrift für Klinische Psychologie und Psychotherapie: Forschung und Praxis 2005; 34: 1-9.

25. Kornor H, Winje D, Ekeberg O, Weisaeth L, Kirkehei I, Johansen K, et al. Early trauma-focused cognitive-behavioural therapy to prevent chronic post-traumatic stress disorder and related symptoms: a systematic review and meta-analysis. BMC Psychiatry 2008; 8: 81.

26. Van Etten M, Taylor S. Comparative efficacy of treatments for post-traumatic stress disorder: a metaanalysis. Clinical Psychology \& Psychotherapy 1998; 5: 126-44.

27. Roberts NP, Kitchiner NJ, Kenardy J, Bisson JI. Systematic review and meta-analysis of multiple-session early interventions following traumatic events. Am J Psychiatry 2009; 166: 293-301.

28. Pae CU, Lim HK, Peindl K, Ajwani N, Serretti A, Patkar $\mathrm{AA}$, et al. The atypical antipsychotics olanzapine and risperidone in the treatment of posttraumatic stress disorder: a meta-analysis of randomized, double-blind, placebo-controlled clinical trials. Int Clin Psychopharmacol 2008; 23: 1-8.

29. Benish SG, Imel ZE, Wampold BE. The relative efficacy of bona fide psychotherapies for treating post-traumatic stress disorder: a meta-analysis of direct comparisons. Clin Psychol Rev 2008; 28: 746-58.

30. Parsons TD, Rizzo AA. Affective outcomes of virtual reality exposure therapy for anxiety and specific phobias: a meta-analysis. J Behav Ther Exp Psychiatry 2008; 39: 250-61.

31. Bisson J, Andrew M. Psychological treatment of posttraumatic stress disorder (PTSD). Cochrane Database Syst Rev 2007: CD003388.

32. Norton PJ, Price EC. A meta-analytic review of adult cognitive-behavioral treatment outcome across the anxiety disorders. J Nerv Ment Dis 2007; 195: 521-31.

33. Adamou M, Puchalska S, Plummer W, Hale AS. Valproate in the treatment of PTSD: systematic review and meta analysis. Curr Med Res Opin 2007; 23: 1285-91.

34. Bisson JI, Ehlers A, Matthews R, Pilling S, Richards D, Turner S. Psychological treatments for chronic posttraumatic stress disorder. Systematic review and metaanalysis. Br J Psychiatry 2007; 190: 97-104.

35. Ipser J, Seedat S, Stein DJ. Pharmacotherapy for posttraumatic stress disorder - a systematic review and meta-analysis. S Afr Med J 2006; 96: 1088-96.

36. Stapleton AB, Lating J, Kirkhart M, Everly GS, Jr. Effects of medical crisis intervention on anxiety, depression, and posttraumatic stress symptoms: a meta-analysis. Psychiatr Q 2006; 77: 231-8.

37. Seidler GH, Wagner FE. Comparing the efficacy of EMDR and trauma-focused cognitive-behavioral therapy in the treatment of PTSD: a meta-analytic study. Psychol Med 2006; 36: 1515-22.

38. Stein DJ, Ipser JC, Seedat S. Pharmacotherapy for post traumatic stress disorder (PTSD). Cochrane Database Syst Rev 2006: CD002795.

39. Cuijpers P, Van Straten A, Smit F. Preventing the inci- 
dence of new cases of mental disorders: a meta-analytic review. J Nerv Ment Dis 2005; 193: 119-25.

40. Bradley R, Greene J, Russ E, Dutra L, Westen D. A multidimensional meta-analysis of psychotherapy for PTSD. Am J Psychiatry 2005; 162: 214-27.

41. Ballenger JC. Remission rates in patients with anxiety disorders treated with paroxetine. J Clin Psychiatry 2004; 65: 1696-707.

42. Deacon BJ, Abramowitz JS. Cognitive and behavioral treatments for anxiety disorders: a review of meta-analytic findings. J Clin Psychol 2004; 60: 429-41.

43. van Emmerik AA, Kamphuis JH, Hulsbosch AM, Emmelkamp PM. Single session debriefing after psychological trauma: a meta-analysis. Lancet 2002; 360: 766-71.

44. Davidson PR, Parker KC. Eye movement desensitization and reprocessing (EMDR): a meta-analysis. J Consult Clin Psychol 2001; 69: 305-16.

45. Adshead G. Psychological therapies for post-traumatic stress disorder. Br J Psychiatry 2000; 177: 144-8.

46. Sherman JJ. Effects of psychotherapeutic treatments for PTSD: a meta-analysis of controlled clinical trials. J Trauma Stress 1998; 11: 413-35.

47. Rose S, Bisson J, Churchill R, Wessely S. Psychological debriefing for preventing post traumatic stress disorder (PTSD). Cochrane Database Syst Rev. 2002: CD000560.

48. Rose S, Bisson J. Brief early psychological interventions following trauma: a systematic review of the literature. J Trauma Stress 1998; 11: 697-710.

49. Lewis SJ. Do one-shot preventive interventions for PTSD work? A systematic research synthesis of psychological debriefings. Aggression and Violent Behavior 2003; 8: 329-43.

50. Taylor TL, Chemtob CM. Efficacy of treatment for child and adolescent traumatic stress. Arch Pediatr Adolesc Med 2004; 158: 786-91.

51. Silverman WK, Ortiz CD, Viswesvaran C, Burns BJ, Kolko DJ, Putnam FW, et al. Evidence-based psychosocial treatments for children and adolescents exposed to traumatic events. J Clin Child Adolesc Psychol 2008; 37: 156-83.
52. Shepherd J, Stein K, Milne R. Eye movement desensitization and reprocessing in the treatment of post-traumatic stress disorder: a review of an emerging therapy. Psychol Med 2000; 30: 863-71.

53. Corner AM, Figgitt DP. Sertraline: A review of its therapeutic use in post-traumatic stress disorder. CNS drugs 2000; 14: 391-407.

54. Mooney P, Oakley J, Ferriter M, Travers R. Sertraline as a treatment for PTSD: a systematic review and metaanalysis. Irish Journal of Psychological Medicine 2004; 21: 100-3.

55. Brewin CR. Systematic review of screening instruments for adults at risk of PTSD. J Trauma Stress 2005; 18: 5362.

56. Rose S, Bisson J, Wessely S. A systematic review of singlesession psychological interventions ('debriefing') following trauma. Psychother Psychosom 2003; 72: 176-84.

57. Brymer MJ, Jacobs A, Layne C. Psychological first aid field operations guide. Washington, DC: National Child Traumatic Stress Network and National Center for PTSD; 2006.

58. Rodriguez JJ, Kohn R. Use of mental health services among disaster survivors. Curr Opin Psychiatry 2008; 21: 370-8.

59. Lowrey W, Evans W, Gower KK, Robinson JA, Ginter PM, McCormick LC, et al. Effective media communication of disasters: pressing problems and recommendations. BMC Public Health 2007; 7: 97.

60. Pan American Health Organization. Protección de la salud mental en situaciones de desastres y emergencias. Serie Manuales y Guías sobre Desastres, № 1. Washington, D.C.; 2002.

61. Clarke L. Panic: myth or reality? Contexts 2002; 1 (3): 21-6.

62. DSM-IV. Manual diagnóstico y estadístico de los trastornos mentales. Primera ed. Barcelona: Masson, S.A.; 1995.

63. Bisson J, Andrew M. Psychological treatment of posttraumatic stress disorder (PTSD). Cochrane Database Syst Rev 2005: CD003388. 